\title{
Ruled Surfaces with Constant Slope Ruling According to Darboux Frame
}

\author{
Ayşe Yavuz*, Fatma Ateş and Yusuf Yaylı
}

\begin{abstract}
The main scope of this paper is to investigate ruled surfaces with constant slope ruling with respect to the given surface according to Darboux Frame. We give necessary and sufficient conditions for these surfaces to be developable. Also, the investigations are observed under some special cases. Especially we obtained that the striction lines and Gaussian curvature of the surfaces.
\end{abstract}

Keywords: Ruled Surface; Surface with Constant Slope; Darboux Frame..

AMS Subject Classification (2020): Primary: 53A04 ; Secondary: 53A05.

*Corresponding author

E-MAIL: ayasar@erbakan.edu.tr E-MAIL: fgokcelik@erbakan.edu.tr E-MAIL: yayli@science.ankara.edu.tr

\section{Introduction}

In differential geometry ruled surface is important surface which is generated by moving a straight line continuously in the space. This surface has many applications in the study of computer aided geometric design. In all studies on curves and surfaces, frame fields play an important role. The most commonly used frames are the Frenet and the Darboux frame. The Darboux frame is build by the velocity of the curve and the normal vector of the surface while the Frenet-Serret frame is constructed from the velocity and the acceleration of the curve. Indicating the derivatives of these frames' vector fields in terms of the vector fields themselves involve some real valued functions. These functions are called the curvature and torsion for the Frenet frame; normal curvature, geodesic curvature and the geodesic torsion for Darboux frame.

Striction curves plays an significant role in the differential geometry and are important for the study of singularities of constant slope surfaces, because it has some interesting specification. The singularities of a ruled surface are located on the striction curve.

\section{Preliminary Results}

In this section, we will give the necessary informations to understand the main subject of the study.

A ruled surface is generated by a straight line acting along a curve. Let $M$ be a surface and let consider a curve $\alpha$ on the surface $M$. In the Frenet frame $\{t, n, b\}$ at each points of the curve $\alpha, t$ is unit tangent vector, $n$ is principal normal vector and $b$ is binormal vector, respectively. The derivative of Frenet frame $\{t, n, b\}$ of the curve $\alpha$ is given as follows.

$$
\begin{aligned}
t^{\prime} & =\kappa n \\
n^{\prime} & =-\kappa t-\tau b \\
b^{\prime} & =-\tau n
\end{aligned}
$$

The functions $\kappa$ and $\tau$, which are respectively determined by the first and third equations in Frenet formula, are called the first and second curvatures of $\alpha(s)$, or respectively the curvature and the torsion. The planes spanned by

Received : 31-10-2019, Accepted : 15-10-2020 
$\{t, n\},\{t, b\}$ and $\{n, b\}$ are called osculating plane, rectifying plane and normal plane, respectively. If the curve $\alpha$ lies on the surface $M$, then another frame is obtained of the curve $\alpha$. This is called Darboux frame and formulized by $\{t, y, N\}$. In this frame $t$ is the unit tangent of the curve, $N$ is the unit normal of the surface $M$ and $y$ is a unit vector. Thus the relations between Frenet and Darboux frames obtained as follows

$$
\begin{aligned}
t^{\prime} & =k_{g} y+k_{n} N, \\
y^{\prime} & =-k_{g} t+t_{r} N, \\
N^{\prime} & =-k_{n} t-t_{r} y,
\end{aligned}
$$

where, $k_{g}$ is the geodesic curvature, defined by equality as follows

$$
k_{g}(s)=\left\langle\alpha^{\prime \prime}(s), y(s)\right\rangle
$$

$k_{n}$ is the normal curvature defined by following equality

$$
k_{n}(s)=\left\langle\alpha^{\prime \prime}(s), N(s)\right\rangle,
$$

and $t_{r}$ is the geodesic torsion of $\alpha(s)$ with following equality

$$
t_{r}(s)=-\left\langle N^{\prime}(s), y(s)\right\rangle .
$$

[4]

Ruled surface with base curve $\alpha$ and generating line $X$ is parameterized as follows

$$
M(u, v)=\alpha(u)+v X(u) .
$$

The equality of the striction curve on the ruled surface is dedicated as follows

$$
\bar{\beta}=\alpha(s)-\frac{\langle\vec{t}, \vec{X}\rangle}{\left\langle\overrightarrow{X^{\prime}}, \overrightarrow{X^{\prime}}\right\rangle} \cdot \vec{X}(s) .
$$

[5]

Ruled surface's Gaussian curvature is defined by

$$
\begin{aligned}
K & =\operatorname{det} S \\
& =-(\langle S(X), Y\rangle)^{2},
\end{aligned}
$$

such that the vector fields $X$ and $Y$ are orthogonal [2].

The standard unit normal vector field $n$ on a surface $M$ can be defined by

$$
n=\frac{\left\langle M_{s}, M_{v}\right\rangle}{\left\|\left\langle M_{s}, M_{v}\right\rangle\right\|}
$$

The first fundamental form characterizes the interior geometry of the surface in a neighborhood of a given point. This means that measurements on the surface can be carried out by means of it. The first fundamental form is given by

$$
I=E d s^{2}+2 F d s d v+G d v^{2}
$$

such that

$$
E=\left\langle M_{s}, M_{s}\right\rangle, \quad F=\left\langle M_{s}, M_{v}\right\rangle \quad G=\left\langle M_{v}, M_{v}\right\rangle
$$

At the same time the second fundamental form

$$
I I=e d s^{2}+2 f d s d v+g d v^{2}
$$


where

$$
e=\left\langle M_{s s}, n\right\rangle, f=\left\langle M_{s v}, n\right\rangle g=\left\langle M_{v v}, n\right\rangle
$$

The Gauss curvature is defined as Weingarten map

$$
K=\operatorname{det} S \text {. }
$$

The Gauss curvatures of a parametrized surface can be computed as

$$
K=\frac{\operatorname{det} I I}{\operatorname{det} I}=\frac{e g-f^{2}}{E G-F^{2}}
$$

[1].

\section{Ruled Surfaces with Constant Slope Ruling According to Darboux Frame}

Let $\phi$ be a ruled surface whose generating lines are given by points on the curve $\alpha$, while in all points they have the constant slope with respect to the tangent planes to the given surface [3]. This surfaces will be called ruled surfaces with constant slope ruling with respect to the given surface.

In this section, we investigate the developable condition for the ruled surfaces with constant slope ruling with respect to the given surface with $\{t, y, N\}$ and we obtain the singular locus of the this surface with Darboux Frame, at the same time we show that singular locus of the constant slope surface is collide with the striction line of the surface. Finally, give some relations and special cases about developable condition.

\subsection{Ruled Surfaces with Constant Slope Ruling with Respect to the $(t, y)$ Planes}

Generating lines of the surface $\phi$ are given by points on the curve $\alpha$ and they have the constant slope $\sigma$ with respect to the $(t, y)$ planes to the curve at every point on the curve $X(s)$. The surface will be called the ruled surfaces with constant slope ruling with respect to the $(t, y)$ planes to a curve.

Direction vector of generating lines of the surface is defined by

$$
X(s)=\sin w(s) t(s)+\cos w(s) y(s)+\sigma N(s)
$$

where the vector $t$ is the direction vector of a tangent to the curve $X, n$ is the direction vector of a normal to the surface and $y=N \times t$ is the direction vector. The surface with constant slope ruling is parametrized by

$$
\phi(s, v)=\alpha(s)+v X(s) .
$$

Example 3.1. Let the curve be a cylindrical helix parametrized by the vector function

$$
\alpha(s)=\left(4 \cos \frac{s}{5}, 4 \sin \frac{s}{5}, 3 \frac{s}{5}\right) .
$$

So the surface parametrization obtained as follows

$$
\begin{aligned}
\phi(s, v)= & \left(4 \cos \frac{s}{5}-v\left(\frac{4}{5} \sin \frac{4 s}{25} \sin \frac{s}{5}+\cos \frac{4 s}{25} \cos \frac{s}{5}-\frac{3}{40} \sin \frac{s}{5}\right),\right. \\
& 4 \sin \frac{s}{5}-v\left(\frac{4}{5} \sin \frac{4 s}{25} \cos \frac{s}{5}-\cos \frac{4 s}{25} \sin \frac{s}{5}-\frac{3}{40} \cos \frac{s}{5}\right), \\
& \left.3 \frac{s}{5}+v\left(\frac{3}{5} \sin \frac{4 s}{25}+\frac{1}{10}\right)\right)
\end{aligned}
$$


The surface for $s \epsilon[0,10], v \epsilon[-10,10]$ and $w(s)=\frac{4}{25} s$ is shown in following figure

Figure 1

Definition 3.1. Surface can be developable ruled surface if the tangent planes are same along the generating lines of the ruled surface. Thus ruled surface is developable if and only if $P_{X}=0$ [2].

Definition 3.2. Dral of the ruled surface is the ratio of the shortest distance between the two generating lines of ruled surface and the angle of between these two lines. So dral of the ruled surfaces is defined by

$$
P_{X}=\frac{\operatorname{det}\left(\frac{d \alpha}{d s}, X, X^{\prime}\right)}{\left\|X^{\prime}\right\|^{2}}
$$

[2].

Theorem 3.1. The necessary and sufficient condition for the ruled surface $\phi(s, v)$ to be developable is given as follows

$$
\cos w(s) .\left(k_{n} \sin w(s)+t_{r} \cos w(s)\right)-\sigma\left(k_{g} \sin w(s)-w^{\prime} \sin w(s)-\sigma t_{r}\right)=0 .
$$

Proof. The surface $\phi$ is developable if and only if $\operatorname{det}\left(t, X, X^{\prime}\right)=0$. So derivative of the direction vector of generating lines of the surface is as follows

$$
\begin{aligned}
X^{\prime}(s)= & \left(\left(w^{\prime}(s)-k_{g}\right) \cos w-\sigma k_{n}\right) t(s) \\
& +\left(-\left(w^{\prime}(s)-k_{g}\right) \sin w-\sigma t_{r}\right) y(s) 3.1 .1 \\
& +\left(k_{n} \sin w(s)+t_{r} \cos w(s)\right) N(s) .
\end{aligned}
$$

And so developable condition for surface $\phi(s, v)$ is given by

$$
\begin{gathered}
\operatorname{det}\left(t, X, X^{\prime}\right)=\cos w(s)\left(k_{n} \sin w(s)+t_{r} \cos w(s)\right)-\sigma\left(k_{g} \sin w(s)-w^{\prime} \sin w(s)-\sigma t_{r}\right), \\
\cos w(s)\left(k_{n} \sin w(s)+t_{r} \cos w(s)\right)-\sigma\left(k_{g} \sin w(s)-w^{\prime} \sin w(s)-\sigma t_{r}\right)=0 .
\end{gathered}
$$

Corollary 3.1. We consider that $w=w_{0}=$ const. Then we get $\cos w_{0}=x_{1}=$ const. and $\sin w_{0}=x_{2}=$ const.So generating lines of the surface is given as follows

$$
X(s)=x_{1} t(s)+x_{2} y(s)+\sigma N(s) .
$$

Let $\alpha$ be an asymptotic line. In that case $k_{n}=0$. Since $\kappa^{2}=k_{n}^{2}+k_{g}^{2}$, we have $k_{g}=\kappa$ and $t_{r}=\tau$. If the surface developable then the main curve is general helix. In fact, from (3.1.1) we obtain as

$$
\frac{\tau}{\kappa}=\frac{\sigma x_{2}}{x_{1}^{2}+\sigma^{2}} \text {. }
$$

Let $\alpha$ be a geodesic curve. In that case $k_{g}=0$. Since $\kappa^{2}=k_{n}^{2}+k_{g}^{2}$, we have $k_{n}=\kappa$ and $t_{r}=\tau$. If the surface developable, then the main curve is general helix. In fact, from (3.1.1) we obtain as

$$
\frac{\tau}{\kappa}=-\frac{x_{1} x_{2}}{2 x_{1}^{2}+\sigma^{2}}
$$

and $\alpha$ is a principal line, the surface is developable if and only if

$$
x_{2}\left(x_{1} k_{n}-\sigma\left(k_{g}\right)\right)=0 .
$$

By using above equation we can say that the surface is developable if and only if $w_{0}=k \pi, k \epsilon Z$ or $x_{1} k_{n}-\sigma k_{g}=0$. 
Corollary 3.2. If

$$
w(s)=\sigma k_{g} d_{s},
$$

such that $\sin w(s) \neq 0$ and $\alpha$ is a principal and asymptotic line, then surface $\phi$ is developable.

Theorem 3.2. The striction line on the surface $\phi(s, v)$ is defined by

$$
\begin{gathered}
\bar{\beta}=\alpha-\frac{-\sigma k_{n}+\left(w^{\prime}(s)-k_{g}\right) \cos w(s)}{\left(w^{\prime}(s)-k_{g}\right)\left(\left(w^{\prime}(s)-k_{g}\right)-2 \sigma k_{n} \cos w(s)+2 \sigma t_{r} \sin w(s)\right)} X(s) . \\
+\left(k_{n} \sin w(s)+t_{r} \cos w(s)\right)^{2}+\sigma^{2}\left(k_{n}^{2}+t_{r}^{2}\right)
\end{gathered}
$$

Proof. By using (3.1.1), we calculate

$$
\left\langle t, X^{\prime}\right\rangle=-\sigma \kappa_{n}+\left(w^{\prime}(s)-k_{g}\right) \cos w(s)
$$

and

$$
\begin{aligned}
\left\langle X^{\prime}, X^{\prime}\right\rangle= & \left(w^{\prime}(s)-k_{g}\right)\left(\left(w^{\prime}(s)-k_{g}\right)-2 \sigma k_{n} \cos w(s)+2 \sigma t_{r} \sin w(s)\right) \\
& +\left(k_{n} \sin w(s)+t_{r} \cos w(s)\right)^{2}+\sigma^{2}\left(k_{n}^{2}+t_{r}^{2}\right)
\end{aligned}
$$

It completes the proof.

Corollary 3.3. If $\alpha$ is an asymptotic line, then $k_{n}=0$. So striction line on the surface defined by

$$
\bar{\beta}=\alpha-\frac{\left(w^{\prime}(s)-k_{g}\right) \cos w(s)}{\left(w^{\prime}(s)-k_{g}\right)\left(w^{\prime}(s)-k_{g}+2 \sigma t_{r} \sin w(s)\right)+\left(t_{r} \cos w(s)\right)^{2}+\sigma^{2} t_{r}^{2}} X(s) .
$$

$\alpha$ is principal line, then $t_{r}=0$ and so striction line is given by

$$
\bar{\beta}=\alpha-\frac{-\sigma \kappa_{n}+\left(w^{\prime}(s)-k_{g}\right) \cos w(s)}{\left(w^{\prime}(s)-k_{g}\right)\left(w^{\prime}(s)-k_{g}-2 \sigma k_{n} \cos w(s)\right)+k_{n} \sin ^{2} w(s)+\sigma^{2} k_{n}^{2}} X(s) .
$$

If $\alpha$ is a geodesic curve, then

$$
\begin{gathered}
\bar{\beta}=\alpha-\frac{-\sigma k_{n}+w^{\prime}(s) \cos w(s)}{w^{\prime}(s)\left(w^{\prime}(s)-2 \sigma k_{n} \cos w(s)+2 \sigma t_{r} \sin w(s)\right)} X(s) . \\
+\left(k_{n} \sin w(s)+t_{r} \cos w(s)\right)^{2}+\sigma^{2}\left(k_{n}^{2}+t_{r}^{2}\right)
\end{gathered}
$$

Corollary 3.4. If $\alpha$ is a geodesic curve and

$$
w(s)=\sigma k_{n} \sec w(s) d s,
$$

then striction line of surface $\phi(s, v)$ is equal to base curve.

Theorem 3.3. The Gaussian curvature of the surface the $\phi(s, v)$ is given by

$$
K=-\frac{(A B v(\sigma-1)+C \cos w(s)-B \cdot \sigma)^{2}}{\left(\left(1+\sigma^{2}\right)\left((1+v A)^{2}+v^{2}\left(B^{2}+C^{2}\right)\right)-((1+v A) \sin w(s)+B \cdot v \cdot \cos w(s)+C \cdot v \cdot \sigma)^{2}\right) \cdot D},
$$

where

$$
\begin{array}{cc}
A= & -\sigma k_{n}+\left(w^{\prime}(s)-k_{g}\right) \cos w(s) \\
B= & -\sigma t_{r}-\left(w^{\prime}(s)-k_{g}\right) \sin w(s) \\
C= & k_{n} \sin w(s)+t_{r} \cos w(s) \\
D= & (B v \sigma-C v \cos w(s))^{2}-(\sigma \cdot(1+v A)-C v \sin w(s))^{2} \\
& +((1+v A) \cos w(s)-B v \sin w(s))^{2}
\end{array}
$$


Proof. The coefficients $E, F$ and $G$ of the first fundamental form of the surface $\phi(s, v)$ are given as follows

$$
\begin{gathered}
E=(1+v A)^{2}+v^{2}\left(B^{2}+C^{2}\right) \\
F=(1+v A) \cdot \sin w(s)+B \cdot v \cdot \cos w(s)+C \cdot v \cdot \sigma \\
G=1+\sigma^{2} .
\end{gathered}
$$

After simple computations, we can easily obtain coefficients $f$ and $g$ of the second fundamental form of $\phi(s, v)$ obtained as follows

$$
\begin{gathered}
f=\frac{A \cdot B \cdot v(\sigma-1)+C \cdot \cos w(s)-B \cdot \sigma}{D} \\
g=0 .
\end{gathered}
$$

The Gauss curvature of a parametrized surface can be computed as follows

$$
K=\frac{e g-f^{2}}{E G-F^{2}}
$$

So we substitute values of coefficients of first and second fundamental form of the $\phi(s, v)$ in above equation, we can find Gauss curvature of $\phi(s, v)$ as follows

$$
K=-\frac{(A B v(\sigma-1)+C \cos w(s)-B \cdot \sigma)^{2}}{\left(\left(1+\sigma^{2}\right)\left((1+v A)^{2}+v^{2}\left(B^{2}+C^{2}\right)\right)-((1+v A) \sin w(s)+B \cdot v \cdot \cos w(s)+C \cdot v \cdot \sigma)^{2}\right) \cdot D} .
$$

\subsection{Ruled Surfaces with Constant Slope Ruling with Respect to the $(N, y)$ Planes}

Surface $\bar{\phi}$ are given by points on the curve $\alpha$ and they have the constant slope $\sigma$ with respect to the $(N, y)$ planes

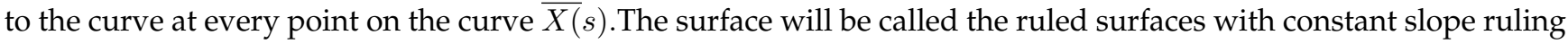
with respect to the $(N, y)$ planes to the a curve. Direction vector of generating lines of the surface is given by

$$
\bar{X}(s)=\sin w(s) N(s)+\cos w(s) y(s)+\sigma t(s) .
$$

The surface with constant slope ruling $\bar{\phi}$ is parametrized by

$$
\bar{\phi}(s, v)=\alpha(s)+v \bar{X}(s) .
$$

Example 3.2. Let the curve parametrized by the vector function

$$
\alpha(s)=\left(\frac{4}{\sqrt{5}} \cos \frac{s \sqrt{5}}{4}, \frac{4}{\sqrt{5}} \sin \frac{s \sqrt{5}}{4}, \frac{r}{\sqrt{5}}\right) .
$$

and the surface parametrized by

$$
\begin{aligned}
\bar{\phi}(s, v)= & \left(\frac{4}{\sqrt{5}} \cos \frac{s \sqrt{5}}{4}+v\left(\sin \frac{s}{2 \sqrt{5}} \cdot \frac{1}{\sqrt{5}} \cos \frac{s \sqrt{5}}{4}-\frac{1}{\sqrt{5}} \cos \frac{s \sqrt{5}}{4} \cos \frac{s}{2 \sqrt{5}}-\frac{1}{4} \sin \frac{s \sqrt{5}}{4}\right)\right. \\
& \frac{4}{\sqrt{5}} \sin \frac{s \sqrt{5}}{4}+v\left(\sin \frac{s}{2 \sqrt{5}} \cdot \frac{1}{\sqrt{5}} \sin \frac{s \sqrt{5}}{4}-\frac{1}{\sqrt{5}} \sin \frac{s \sqrt{5}}{4} \cos \frac{s}{2 \sqrt{5}}+\frac{1}{4} \cos \frac{s \sqrt{5}}{4}\right) \\
& \frac{4}{\sqrt{5}}+v\left(\sin \frac{s}{2 \sqrt{5}}+\frac{1}{\sqrt{5}}\right)
\end{aligned}
$$


The surface for $s \epsilon[0,3 \sqrt{5} \pi], v \epsilon[-5,5]$ and $w(s)=\frac{s}{2 \sqrt{5}}$ is shown in following figure

Figure 2

Theorem 3.4. The ruled surface $\bar{\phi}(s, v)$ is developable if and only if

$$
-w^{\prime}(s)-t_{r}+\sigma\left(k_{g} \sin w(s)-k_{n} \cos w(s)\right)=0
$$

Proof. If we take derivative of the direction vector of generating lines of the surface $\bar{X}$ with respect to the parameter $s$, then we get

$$
\begin{aligned}
\bar{X}^{\prime}(s)= & \left.\left(w^{\prime}(s)+t_{r}\right) \cos w(s)+k_{n} \sigma\right) N(s)+\left(-\left(w^{\prime}(s)+t_{r}\right) \sin w(s)+k_{g} \sigma\right) y(s) \\
& +\left(-k_{n} \sin w(s)-k_{g} \cos w(s)\right) t(s) .
\end{aligned}
$$

We easily find that

$$
\begin{aligned}
\operatorname{det}\left(t, \bar{X}, \bar{X}^{\prime}\right)= & \sin w(s)\left(\sin w(s)\left(-t_{r}-w^{\prime}(s)\right)+k_{g} \sigma\right)-\cos w(s)\left(\cos w(s)\left(w^{\prime}(s)+t_{r}\right)+k_{n} \sigma\right) \\
& -\left(w^{\prime}(s)+t_{r}\right)+\sigma\left(k_{g} \sin w(s)-k_{n} \cos w(s)\right) .
\end{aligned}
$$

So developable condition for surface $\bar{\phi}(s, v)$ is given by

$$
-w^{\prime}(s)-t_{r}+\sigma\left(k_{g} \sin w(s)-k_{n} \cos w(s)\right)=0 .
$$

Corollary 3.5. We consider that $\cos w(s)=x_{1}=$ const and $\sin w(s)=x_{2}=$ const.

$$
X(s)=x_{1} N(s)+x_{2} y(s)+\sigma t(s) .
$$

Let $\alpha$ is an asymptotic line, the surface is developable if and only if base curve is general helix by

$$
\frac{\tau}{\kappa}=\sigma x_{2} .
$$

Similarly, $\alpha$ is a geodesic curve, the surface is developable if and only if base curve is general helix by

$$
\sigma x_{1}=\frac{\tau}{\kappa}
$$

Theorem 3.5. The striction line on the surface $\bar{\phi}(s, v)$ is given by

$$
\bar{\beta}=\alpha-\left(\begin{array}{c}
-k_{n} \sin w(s)-k_{g} \cos w(s) \\
\frac{\left(w^{\prime}(s)+t_{r}\right)^{2}+2 \sigma\left(w^{\prime}(s)+t_{r}\right)\left(k_{n} \cos w(s)-k_{g} \sin w(s)\right)}{+\left(k_{n} \sin w(s)+k_{g} \cos w(s)\right)^{2}+\sigma^{2}\left(k_{n}^{2}+k_{g}^{2}\right)}
\end{array}\right) X(s) .
$$


Corollary 3.6. If $\left(w^{\prime}(s)+t_{r}\right)=0$, then

$$
\bar{\beta}=\alpha+()-k_{n} \sin w(s)-k_{g} \cos w(s)\left(k_{n} \sin w(s)+k_{g} \cos w(s)\right)^{2}+\sigma^{2}\left(k_{n}^{2}+k_{g}^{2}\right) \cdot X(s) .
$$

If $\alpha$ is an asymptotic curve, then $k_{n}=0$. So striction line on the surface defined by

$$
\bar{\beta}=\alpha-\left(\begin{array}{c}
-k_{g} \cos w(s) \\
\left(w^{\prime}(s)+t_{r}\right)^{2}+2 \sigma\left(w^{\prime}(s)+t_{r}\right)-k_{g} \sin w(s) \\
+\left(k_{g} \cos w(s)\right)^{2}+\sigma^{2} k_{g}^{2}
\end{array}\right) X(s) .
$$

$\alpha$ be a principal line, then $t_{r}=0$ and so striction line is

$$
\bar{\beta}=\alpha(s)-\left(\frac{-k_{n} \sin w(s)-k_{g} \cos w(s)}{\frac{w^{\prime}(s)^{2}+2 \sigma w^{\prime}(s)\left[k_{n} \cos w(s)-k_{g} \sin w(s)\right]}{+\left[k_{n} \sin w(s)+k_{g} \cos w(s)\right]^{2}+\sigma^{2}\left(k_{n}^{2}+k_{g}^{2}\right)}}\right) X(s) .
$$

If $\alpha$ is a geodesic curve, then

$$
\bar{\beta}=\alpha(s)-\left(\begin{array}{c}
-k_{n} \sin w(s) \\
\begin{array}{c}
\left(w^{\prime}(s)+t_{r}\right)^{2}+2 \sigma\left(w^{\prime}(s)+t_{r}\right) k_{n} \cos w(s) \\
+\left[k_{n} \sin w(s)\right]^{2}+\sigma^{2} k_{n}^{2}
\end{array}
\end{array}\right) X(s) .
$$

Theorem 3.6. The Gaussian curvature of the surface $\bar{\phi}(s, v)$ is given by

$$
K=-\frac{\left(\left(w^{\prime}(s)+t_{r}\right)\left(\sigma\left(b \cdot \cos ^{2} w(s)+a \sin ^{2} w(s)\right)+c\right)-c(1+v c)\right)^{2}}{\left(v\left(^{2}\left(a^{2}+b^{2}+c^{2}\right)+1-2 v c .\right)\left(1+\sigma^{2}\right)-\sigma^{2} \cdot(c(1-v)+1)^{2}\right) \cdot d},
$$

where

$$
\begin{aligned}
a & \left.=\left(w^{\prime}(s)+t_{r}\right) \cos w(s)+k_{n} \sigma\right) \\
b & =\left(-\left(w^{\prime}(s)+t_{r}\right) \sin w(s)+k_{g} \sigma\right) \\
c & =\left(k_{n} \sin w(s)+k_{g} \cos w(s)\right. \\
d & =(\sigma . b-c(1+v c) \cos w(s))^{2}+(\sigma \cdot a-c(1+v c) \sin w(s))^{2}+\left(w^{\prime}(s)+t_{r}\right)^{2} .
\end{aligned}
$$

\subsection{Ruled Surfaces with Constant Slope Ruling with Respect to the $(t, N)$ Planes}

Generating lines of the surface $\widetilde{\phi}$ are given by points on the curve $\alpha$ and they have the constant slope $\sigma$ with respect to the $(t, N)$ planes to the curve at every point on the curve $\widetilde{X}(s)$. The surface will be called the ruled surfaces with constant slope ruling with respect to the $(t, N)$ planes to the a curve. Direction vector of generating lines of the surface is obtained as follows

$$
\tilde{X}(s)=\sin w(s) t(s)+\cos w(s) N(s)+\sigma y(s) .
$$

The surface with constant slope ruling $\widetilde{\phi}$ is parametrized by

$$
\widetilde{\phi}(s, v)=\alpha(s)+v(\sin w(s) t(s)+\cos w(s) N(s)+\sigma y(s)) .
$$

Theorem 3.7. The surface $\widetilde{\phi}(s, v)$ is developable if and only if

$$
\cos w(s)\left(\sin w(s) k_{g}-\cos w(s) t_{r}\right)+\sigma\left(-w^{\prime}(s)+k_{n} \sin w(s)+\sigma t_{r}\right)=0 .
$$

Proof. By taking derivative of the direction vector of generating lines of the surface $\widetilde{X}$ with respect to parameter $s$, then we get

$$
\begin{aligned}
\tilde{X}^{\prime}(s)= & t(s)\left[\left(w^{\prime}(s)-k_{n}\right) \cos w(s)-\sigma k_{g}\right] \\
& +N(s)\left[-\left(w^{\prime}(s)-k_{n}\right) \sin w(s)+\sigma t_{r}\right] \\
& +y(s)\left[\sin w(s) . k_{g}-t_{r} \cos w(s)\right]
\end{aligned}
$$




$$
\operatorname{det}\left(t, \tilde{X}, \tilde{X}^{\prime}\right)=\cos w(s)\left[\sin w(s) k_{g}-\cos w(s) t_{r}\right]+\sigma\left[-\left(w^{\prime}(s)-k_{n}\right) \sin w(s)+\sigma t_{r}\right] .
$$

So developable condition for surface $\bar{\phi}(s, v)$ is given by

$$
\cos w(s)\left(\sin w(s) k_{g}-\cos w(s) t_{r}\right)+\sigma\left(-\left(w^{\prime}(s)-k_{n}\right) \sin w(s)+\sigma t_{r}\right)=0
$$

Corollary 3.7. We consider that $\cos w(s)=x_{1}=$ const and $\sin w(s)=x_{2}=$ const.

$$
X(s)=x_{1} N(s)+x_{2} y(s)+\sigma t(s) .
$$

Let $\alpha$ is an asymptotic line, the surface is developable if and only if base curve is general helix by

$$
\frac{\tau}{\kappa}=\frac{x_{1} x_{2}}{x_{1}^{2}+\sigma^{2}}
$$

Similarly, $\alpha$ is a geodesic curve, the surface is developable if and only if the base curve is general helix by

$$
\frac{\tau}{\kappa}=\frac{\sigma x_{2}}{x_{1}^{2}+\sigma^{2}}
$$

and $\alpha$ is a principal line, the surface is developable if and only if

$$
x_{2}\left(x_{1} k_{g}+\sigma k_{n}\right)=0
$$

so

$$
\frac{k_{n}}{k_{g}}=\frac{-x_{1}}{\sigma}
$$

\section{Corollary 3.8. If}

$$
w(s)=k_{n} d_{s},
$$

such that $\sin w(s) \neq 0$ and $\alpha$ is a principal and geodesic line, then surface $\widetilde{\phi}$ is developable.

Theorem 3.8. The striction line on the surface $\widetilde{\phi}$ is obtained as follows

$\bar{\beta}=\alpha-()\left(w^{\prime}(s)-k_{n}\right) \cos w(s)-\sigma k_{g} \begin{gathered}\left(w^{\prime}(s)-k_{n}\right)^{2}+\sigma^{2}\left(k_{g}^{2}+t_{r}^{2}\right)-2 \sigma\left(w^{\prime}(s)-k_{n}\right)\left(k_{g} \cos w(s)+t_{r} \sin w(s)\right) \\ +\left(\left(\sin w(s) k_{g}-t_{r} \cos w(s)\right)^{2}\right.\end{gathered}$

Corollary 3.9. At the same time both $w^{\prime}(s)=k_{n}$, striction line on the surface is given by

$$
\bar{\beta}=\alpha-()-\sigma k_{g} \sigma^{2}\left(k_{g}^{2}+t_{r}^{2}\right)+\left(\left(\sin w(s) k_{g}-t_{r} \cos w(s)\right)^{2} \cdot \widetilde{X}(s) .\right.
$$

Corollary 3.10. Let $\cos w(s)=x_{1}=$ const and $\sin w(s)=x_{2}=$ const.If

$$
\frac{k_{g}}{k_{n}}=\frac{-x_{1}}{\sigma}
$$

then striction line on the surface $\widetilde{\phi}$ equal to base curve of the surface the $\widetilde{\phi}$.

Corollary 3.11. It has been shown in [1], singular locus of the constant slope surfaces $\phi(s, v), \widetilde{\phi}(s, v), \bar{\phi}(s, v)$ are striction lines of these surfaces.

Theorem 3.9. The Gaussian curvature of the surface $\widetilde{\phi}(s, v)$ is given by

$$
K=-\frac{((1+v P)(R \sigma-S \cos w(s)+\sigma R v+v \cdot S \cdot \sigma)-2 v R S \sin w(s))^{2}}{\left((1+v P)^{2}+v^{2}\left(R^{2}+S^{2}\right)\right)\left(1+\sigma^{2}\right) \cdot T},
$$

where

$$
\begin{aligned}
P & =\left(w^{\prime}(s)-k_{n}\right) \cos w(s)-\sigma k_{g} \\
R & =-\left(w^{\prime}(s)-k_{n}\right) \sin w(s)+\sigma t_{r} \\
S & =\sin w(s) \cdot k_{g}-t_{r} \cos w(s) \\
T & =(R \sigma-S \cos w(s))^{2}+(\sigma(1+v P)-S \sin w(s))^{2}+((1+v P) \sigma-R \sin w(s))^{2}
\end{aligned}
$$




\section{References}

[1] Do Carmo, M. P.: Differential Geometry of Curves and Surfaces. Prentice-Hall, ISBN 0-13-212589- 7 (1976).

[2] Hacısalihoğlu H.H.: Differential Geometry. Ankara, Faculty of Science Publ. (2000).

[3] Malecek K, Szarka J, Szarková D : Surfaces With Constant Slope and Their Generalization. J. Polish Society Geometry Eng. Graphics, 19:67-77 (2009).

[4] Sabuncuoğlu A. : Differential Geometry, Nobel Publication. ISBN978-975-591-237-0. Ankara (2010).

[5] Papageorgiou S. G., Aspragathos N. A.: Rational ruled surfaces construction by interpolating dual unit vectors representing lines. In: International Conference on Computer graphics, Visualization and Computer Vision, Pilzen, Czech Republic, 30 January-3 February 2006. Pilzen: WSCG, University of West Bohemia ( 2006).

\section{Affiliations}

AYŞE YAVUZ

AdDress: Necmettin Erbakan University, Department of Mathematics and Science Education, 42090, KonyaTurkey.

E-MAIL: ayasar@erbakan.edu.tr

ORCID ID:0000-0002-0469-3786

FATMA ATEŞ

ADDRESS: Necmettin Erbakan University, Department of Mathematics-Computer Sciences, 42090, Konya-Turkey. E-MAIL: fgokcelik@erbakan.edu.tr

ORCID ID:0000-0002-3529-1077

YUSUF YAYLI

AdDRESS: Ankara University, Department of Mathematics, 06560, Ankara-Turkey.

E-MAIL: yayli@science.ankara.edu.tr

ORCID ID:0000-0003-4398-3855 